\title{
Digital Health during COVID-19: Informatics Dialogue with the World Health Organization
}

\author{
Sabine Koch ${ }^{*}$, William R. Hersh', Riccardo Bellazzi ${ }^{3,4}$, Tze Yun Leong ${ }^{5}$, Moctar Yedaly ${ }^{6}$, \\ Najeeb Al-Shorbaji ${ }^{7}$ \\ 1 Health Informatics Centre, Department of Learning, Informatics, Management and Ethics, \\ Karolinska Institutet, Stockholm, Sweden \\ 2 Department of Medical Informatics \& Clinical Epidemiology, School of Medicine, Oregon Health \& \\ Science University, Portland, OR, USA \\ 3 Department of Electrical, Computer and Biomedical Engineering, University of Pavia, Italy \\ ${ }^{4}$ IRCCS ICS Maugeri Pavia, Italy \\ 5 Department of Computer Science, School of Computing, National University of Singapore and Al \\ Singapore, Singapore \\ 6 Information Society Division, African Union Commission, Addis Ababa, Ethiopia \\ 7 eHealth Development Association, Amman, Jordan
}

\section{Summary \\ Background: On December 16, 2020 representatives of the International Medical Informatics Association (IMIA), a Non-Gov- ernmental Organization in official relations with the World Health Organization (WHO), along with its International Academy for Health Sciences Informatics (IAHSI), held an open dialogue with WHO Director General (WHO DG) Tedros Adhanom Ghebreyesus about the opportunities and challenges of digital health during the COVID-19 global pandemic. \\ Objectives: The aim of this paper is to report the outcomes of the dialogue and discussions with more than 200 participants representing different civil society organizations (CSOs).}

\begin{abstract}
Methods: The dialogue was held in form of a webinar. After an initial address of the WHO DG, short presentations by the panelists, and live discussions between panelists, the WHO DG and WHO representatives took place. The audience was able to post questions in written. These written discussions were saved with participants' consent and summarized in this paper.

Results: The main themes that were brought up by the audience for discussion were: (a) opportunities and challenges in general; (b) ethics and artificial intelligence; (c) digital divide; (d) education. Proposed actions included the development of a roadmap based on the lessons learned from the COVID-19 pandemic.
\end{abstract}

Conclusions: Decision making by policy makers needs to be evidence-based and health informatics research should be used to support decisions surrounding digital health, and we further propose next steps in the collaboration between IMIA and WHO such as future engagement in the World Health Assembly.

\section{Keywords}

COVID-19, Medical Informatics, Artificial Intelligence, Global Health, World Health Organization

Yearb Med Inform 2021:13-6

http://dx.doi.org/10.1055/s-0041-1726480

\section{Introduction}

During the COVID-19 worldwide pandemic, the essential role of biomedical and health informatics to improve preparedness, surveillance, and continued delivery of evidence-based best practices became evident. The International Medical Informatics Association (IMIA) is the world body for biomedical and health informatics, providing informatics leadership and expertise with the world-wide vision of improving the health of the world population. The International Academy of Health Sciences Informatics (IAHSI) is the honorific society of IMIA and consists of elected Fellows who represent many of the world's leading experts in health and biomedical informatics. Recognizing the potential impact of IMIA and IAHSI to contribute towards combat of the physical pandemic as well as manage the concurrent "infodemic", the IAHSI Committee on Health Informatics in Pandemics addressed the World Health Organization (WHO) in a statement on the use of informatics in pandemic situations. In this statement, the WHO was invited to collaborate with IMIA/IAHSI specifically in four areas: artificial intelligence (AI), virtual care, patient-centered health data sharing, and data and information quality [1].

\footnotetext{
* The authors represented IMIA/IAHSI in the dialogue session in different roles:

SK - IMIA President and corresponding author

WBH - IAHSI President

RB - former IMIA Vice President and data analytics expert

TYL - former IMIA Vice President and AI expert

MY - representing the African Union Commission

NAS - current IMIA Vice President, IAHSI Committee Chair, and panel moderator
} 
When the WHO issued a call for proposals to organize dialogues between civil society organizations (CSO) and the WHO Director General on different topics of interest for the civil society related to COVID-19 response, IMIA/IAHSI applied for a dialogue on digital health. This dialogue about the opportunities and challenges of digital health during the COVID-19 global pandemic was held on December 16, 2020.

The dialogue, in form of a webinar, consisted of short introductions by the WHO Director General (DG) and the authors, and was followed by more than 200 participants posing numerous questions and engaging in discussions. This paper provides a summary of the presentations and discussions and proposes further actions.

\section{Aim of the IMIA/IAHSI - WHO DG Dialogue}

During COVID-19, health informatics and the use of information and communication technology in general has become the norm for health care delivery. Use of telehealth and telemedicine, contact tracing, health data processing, and mapping and use of social media to fight misinformation (infodemics) are some examples of the way digital health has been used recently. Absence of policies, strategies, trained personnel, and appropriate digital health applications has resulted in much of the unpreparedness in many countries in handling the pandemic.

IMIA as an international association represents health and medical informatics professionals and academics from around the world, who are experts in adequate management of data and information. These professionals offer knowledge and propose solutions, but seek guidance at policy level and require a platform to express their views at global level to support health systems worldwide. Given the status of WHO as the global body in charge of health policy setting and evidence-building, it becomes imperative to engage different stakeholders via WHO leadership.

The dialogue with the WHO DG was an opportunity to reach out to different actors within WHO and global CSOs pursuing the common aim of strengthening health systems. This dialogue aimed to refocus the WHO global leadership role in this area, as digital health constitutes a major component in health systems. The objectives of the dialogue were:

- to establish a higher profile of digital health to support health systems;

- to create awareness of the value of digital health based on the experience of experts in the field;

- to identify challenges facing digital health: technology infrastructure, organizational, legal and ethical issues.

\section{Conducting the IMIA/IAHSI - WHO DG Dialogue}

WHO Director General Dr. Tedros Ghebreyesus opened the dialogue session. In his opening address [2], he highlighted the evolving potential of digital technologies for transforming the health of entire populations and promoted a proactive and inclusive approach to protect safety, human rights and privacy. He further stated the co-creation of the digital transformation of health and the importance of bringing together the global digital health community to implement the WHO Global Strategy on Digital Health.

The session moderator Dr. Najeeb Al-Shorbaji gave a short introduction into the topic and the objectives of the session, highlighting the importance of the topic and the potential contributions IMIA as an organization in official relations to $\mathrm{WHO}$ could make to further strengthen the relationship and contributing to the implementation of the Global Strategy on Digital Health.

IMIA President Dr. Sabine Koch presented IMIA as a global organization, a federation comprising 57 member societies and including representatives from roughly 100 countries around the world. She reported how the COVID-19 pandemic has accelerated the use of digital health, as well as having emphasized the need for high quality data capture, timely management and ethical use of the data. She further described the need to guide the implementation of WHO strategies and policies in a national context, and to follow up on the effects of the implementation.
IAHSI President Dr. William Hersh represented the 179 Fellows of IMIA's honorific society and gave an overview of the competence and knowledge available in this group regarding research and education worldwide. He also highlighted some of the ongoing IAHSI activities in form of panels for informatics in standards and interoperability, AI, and computational knowledge. Dr. Hersh also highlighted the critical role that many IMIA members play in academic institutions, leading research and education of the next generation of informatics professionals and leaders who will advance the field.

Dr. Riccardo Bellazzi spoke on behalf the 26 IMIA Working and Special Interest Groups, thematically covering all areas of biomedical and health informatics. He mentioned the initiatives of the Working Groups carried on during the pandemic [3], and he presented the 4CE initiative [4] for the clinical characterization of COVID patients on the basis of electronic health record data. Finally, he highlighted the natural partnership between the IMIA and WHO, since IMIA feeds the WHO strategic vision about digital health with knowledge and wisdom of its scientists and educators.

Dr. Tze-Yun Leong shared her experiences about the use of AI in Singapore during the COVID-19 pandemic. She explained how $\mathrm{AI}$ and digital technologies can contribute to the care continuum, especially in rapid diagnosis, process management, vaccine development and distribution, and remote consultation for telehealth and mental health. She summarized the challenges and solutions of introducing technology-enabled contact tracing in practice. She also highlighted the importance of building trust to facilitate $\mathrm{AI}$ adoption through responsible innovation with relevant ethical, governance, regulatory considerations, digital competency, and risk communication to support resilient pandemic response and recovery.

Mr. Moctar Yedely reported from his long-standing work in setting up and managing telehealth infrastructures and networks in the African Union, and the importance of local adaptations.

In between the short presentations by the panelists, live discussions between panelists, the WHO DG and WHO representatives took place. The audience was able to post ques- 
tions in the chat and/or question/answering module of the webinar. The moderator took up several posts by the audience for live discussion. The written discussions were saved with participants' consent and analyzed to write this paper. The webinar as such has not been recorded.

\section{Results and Discussion}

The analysis of the written discussions is presented according to different themes that were brought up by the audience.

\subsection{Opportunities and Challenges in General}

Many opportunities with digital health and informatics were mentioned such as developing structures and processes for capturing high quality data, the use of AI for early detection and surveillance of pandemics, and virtual care and remote triage to lessen the strain on health systems.

Several challenges were also brought up such as securing the ethical use of AI, securing the quality of healthcare information, avoiding infodemics, and to better understand and respond to local needs, especially in low and middle-income countries (LMIC) settings. Concerns for human rights by digital health surveillance during COVID-19 were referred to [5], and strong governance requested to secure the potentials of digitalization for health while also addressing the concerns.

\subsection{Ethics and Al}

Several ethical questions were raised. Some participants believed that individual privacy should not be maintained if one's infectious disease impacts others, and data about individual health need to be shared. Others promoted a demonstration project by IAHSI members about COVID-19 data that can be ethically and securely shared without impeding on individual privacy. Sharing (and selling) personal data for commercial purposes was seen as a serious threat.
Several participants seemed to agree that we need to accelerate the sharing of codified clinical knowledge for COVID-19 research and care around the world and the use of open, findable, accessible, interoperable and reusable (FAIR) COVID-19 data was recommended [6], under close surveillance of ethical, legal, and social implications.

WHO has been conducting webinars on ethical and legal issues of digital health and plans to issue guidelines on these, especially in the context of AI. WHO, together with partner organizations such as the International Telecommunication Union (ITU), has convened expert panels and working groups to deliberate on and formulate best-practice guidelines for Ethics and Governance of AI in Health, and Regulatory Considerations on AI for Health. Some IMIA/IAHSI informatics experts are serving on these panels. As AI can produce novel and unpredicted solutions, even "transparent" AI can be very difficult to reason about. This raises an ethical issue: can patients give truly informed consent if neither they nor the healthcare provider can fully understand how AI is influencing healthcare?

We urgently need more evidence about AI solutions and their effects. Can we create trustable and reusable $\mathrm{AI}$ and data analytics code and share it openly together with implementation guidelines? WHO should provide governance for the responsible use similar to those already published for parts of the AI field [7].

\subsection{Digital Divide}

Digital Health requires Internet access and a device to access and use the Internet. Disparities exist, not only in LMICs. High income countries also have digital divides, creating challenges depending on individual socio-economic, geographic, educational and health backgrounds. Can WHO and partners such as ITU help make true global access to the Internet a goal? IMIA can help to increase e-health literacy through education as well as promote inclusive design of digital health.

We also need to bridge the digital divide and reduce health disparities regarding COVID-19 data collection and reporting.
Whereas WHO can work towards universal access to vaccines, the IAHSI panel on standards for digital health works on common structures for vaccination records. This will lay the ground for vaccine safety surveillance. Documentation of post COVID-19 complications was discussed as another important area. To build up the knowledge base, high quality, structured documentation in EHRs and/or registries is necessary as the pandemic pointed out severe limitations in EHR data quality.

\subsection{Education}

Health literacy and e-health literacy are critical to move forward. Education and prevention of infodemics of inaccurate health information can contribute to improve health literacy throughout the world. The question whether WHO would consider making a third attempt at setting up a dot health (.health) top level domain for trusted sources of health information was raised.

IMIA-affiliated academic institutions could directly participate through providing education for many audiences from health care to informatics professionals, and also collaborating from others in other academic organizations through the WHO Academy [8], an effort to disseminate health-related learning worldwide. This would be one way to help to disseminate the knowledge around best practices in informatics, data science, digital health, and related areas.

Digital health is one tool towards achieving global health goals, and the digital health community needs to integrate more fully with other disciplines and movements such as Healthcare Information For All (HIFA) [9]. Communities of Practice (CoPs) have an important role here to ensure alignment with wider learning and best practice.

\section{Proposed Actions for IMIA/IAHSI \& WHO}

Based on the lessons learned from the COVID-19 pandemic, WHO in partnership with IMIA should create a roadmap including: 
1) Creation of a digital health library of evidence-based, reusable digital health approaches that support: (1) vaccine development and monitoring; (2) digital health care practices to support social distancing; (3) day-to-day healthcare during the current pandemic; (4) pandemic management; and (5) citizen uptake of evidence-based health information and digital health, thereby supporting evidence-based citizen decision making regarding health;

2) Disseminating knowledge and education through IMIA academic members and collaboration with the WHO Academy;

3) Furthering stakeholder engagement: various communities need to be brought together to enable effective digital health globally. Most importantly, support of the governments and the engagement and commitment of every country are needed. WHO as a global organization can make that happen.

\section{Conclusion}

Decision making by policy makers needs to be evidence-based and health informatics research should be used to support decisions surrounding digital health. IMIA and its international members have deep knowledge and experience of research, education, and day-to-day management in different areas of digital health, and we are eager and willing to contribute with our expertise.

More specifically, we propose the development of a research programme that aims to collect, synthesize and present evidence how and under which circumstances digi- tal health can contribute to equity, reduce cost and provide efficiency in health care delivery. This effort should include a focus on the role of informatics professionals in developing and leading such efforts. IMIA/ IAHSI can contribute with evidence building and knowledge dissemination, and through its society members influence policy making in many countries together with WHO.

Proposed next steps in the IMIA-WHO collaboration include engagement of the IMIA working groups and IAHSI task forces in writing of WHO policy documents in the area of digital health such as the guidance on ethics and governance of AI for health, as well as to inform member state representatives and to host a side event on digital health at the World Health Assembly.

\section{Acknowledgment}

The authors thank the World Health Organization and IMIA CEO Elaine Huesing for organizing this dialogue, and the WHO DG and his team, as well as all online participants for valuable input and fruitful discussions.

\section{References}

1. Al-Shorbaji N, Haux R, Koch S. Statement from the International Academy for Health Sciences Informatics (IAHSI), the Academy of the International Medical Informatics Association (IMIA) to the Director General of the WHO on the Use of Informatics in Pandemic Situations [Internet]. 2020 [cited 2021 Jan 31]. Available from: https:// imia-medinfo.org/wp/statement-from-the-international-academy-for-health-sciences-informatics-iahsi-the-academy-of-the-international-medical-informatics-association-imia-to-the-directorgeneral-of-the-who-on-the-use/

2. Ghebreyesus TA. DG CSO dialogue "Digital
Health during COVID-19: Opportunities and Challenges" for the International Medical Informatics Association (IMIA) and IMIA's International Academy for Health Sciences Informatics (IAHSI) (who.int). Dec 16, 2020 [cited 2021 Jan 31].

3. Fernandez-Luque L, Kushniruk AW, Georgiou A, Basu A, Petersen C, Ronquillo C, et al. Evidence-based health informatics as the foundation for the COVID-19 response: a joint call for action. Methods Inf Med. Forthcoming 2021.

4. Brat GA, Weber GM, Gehlenborg N, Avillach P, Palmer NP, Chiovato L, et al. International electronic health record-derived COVID-19 clinical course profiles: the 4CE consortium. NPJ Digit Med. 2020 Aug 19;3:109.

5. Sekalala S, Dagron S, Forman L, Meier BM. Analyzing the Human Rights Impact of Increased Digital Public Health Surveillance during the COVID-19 Crisis. Health Hum Rights 2020;22(2): 7-20.

6. Wilkinson MD, Dumontier M, Aalbersberg IJJ, Appleton G, Axton M, Baak A, et al. The FAIR Guiding Principles for scientific data management and stewardship. Sci Data 2016;3:160018. Available from: https://doi.org/10.1038/sdata.2016.18

7. World Economic Forum 2020. Chatbots RESET: A Framework for Governing Responsible Use of Conversational AI in Healthcare [cited $2021 \mathrm{Feb} 7$ ]. Available from: https://www.weforum.org/reports/ chatbots-reset-a-framework-for-governing-responsible-use-of-conversational-ai-in-healthcare

8. WHO Academy. Revolutionizing lifelong learning in health [cited 2021 Feb 7]. Available from: https:// www.who.int/about/who-academy

9. Healthcare Information for All [cited 2021 Feb 7]. Available from: http:/hifa.org/

Correspondence to:

Prof. Dr. Sabine Koch

Health Informatics Centre

Department of Learning, Informatics, Management and Ethics

Karolinska Institutet

Tomtebodavägen 18a

17177 Stockholm

Sweden

E-mail: sabine.koch@ki.se

ki.se/hic 